\title{
Quantum State Diffusion and Time Correlation Functions
}

\author{
Todd A. Brun \\ Physics Department, Queen Mary and Westfield College, \\ University of London, London E1 4NS, England \\ and \\ Nicolas Gisin \\ Group of Applied Physics \\ University of Geneva, 1211 Geneva 4, Switzerland
}

November 6, 2018

\begin{abstract}
In computing the spectra of quantum mechanical systems one encounters the Fourier transforms of time correlation functions, as given by the quantum regression theorem for systems described by master equations. Quantum state diffusion (QSD) gives a useful method of solving these problems by unraveling the master equation into stochastic trajectories; but there is no generally accepted definition of a time correlation function for a single QSD trajectory. In this paper we show how QSD can be used to calculate these spectra directly; by formally solving the equations which arise, we arrive at a natural definition for a two-time correlation function in QSD, which depends explicitly on both the stochastic noise of the particular trajectory and the time of measurement, and which agrees in the mean with the ensemble average definition of correlation functions.
\end{abstract}




\section{Introduction}

In quantum optics, a common experimental situation involves a system in a cavity which is monitored by measuring the spectrum of output photons. In order to compare theory to experiment it is necessary to calculate this output spectrum.

What is commonly found is that the intensity of the output spectrum is given by the Fourier transform of two-time correlation functions of system variables [1]. In the case of a system isolated from its environment (apart from the measurement process itself), these correlation functions are simply the expectation values of products of Heisenberg operators at different times, e.g., $\left\langle q\left(t_{2}\right) q\left(t_{1}\right)\right\rangle$.

As it is difficult to completely isolate a system from the environment, this approach does not always succeed. In the case of a quantum open system interacting continually with an external reservoir, one instead describes the system by a master equation, which - within the Markov approximation can be written in Lindblad form:

$$
\dot{\rho}=-i[H, \rho]+\sum_{m}\left(L_{m} \rho L_{m}^{\dagger}-\frac{1}{2}\left\{L_{m}^{\dagger} L_{m}, \rho\right\}\right) .
$$

The $L_{m}$ are a set of environment operators which give the collective effects of the environment, and we have taken $\hbar=1$.

The density operator $\rho$ gives the probability for the expected outcomes of measurements on the system. In this case, one can still calculate output spectra, and find Fourier transforms of time correlation functions; these are no longer the expectation values of products of Heisenberg operators in the system Hilbert space alone, but are more complicated objects whose form is given by the quantum regression theorem [2, 14, 淉.

Unfortunately, for complicated systems equation (1) can be very difficult to solve either analytically or numerically. In that case, it is often advantageous to consider an unraveling of the master equation into individual quantum trajectories, each one represented by a single state at every moment in time. One of these unraveling techniques is quantum state diffusion (QSD) [5]. In this, the normalized vector $|\psi\rangle$ representing the pure state of the system evolves according to the QSD equation:

$$
d|\psi\rangle=-i H|\psi\rangle d t+\sum_{m}\left(\left\langle L_{m}^{\dagger}\right\rangle L_{m}-\frac{1}{2} L_{m}^{\dagger} L_{m}-\frac{1}{2}\left\langle L_{m}^{\dagger}\right\rangle\left\langle L_{m}\right\rangle\right)|\psi\rangle d t
$$




$$
+\sum_{m}\left(L_{m}-\left\langle L_{m}\right\rangle\right)|\psi\rangle d \xi_{m} .
$$

This is an Itô stochastic differential equation, in which the $d \xi_{m}$ represent independent complex Wiener processes. These satisfy

$$
M\left(d \xi_{m}\right)=M\left(d \xi_{m} d \xi_{n}\right)=0, \quad M\left(d \xi_{m}^{*} d \xi_{n}\right)=\delta_{m n} d t,
$$

where $M$ represents an ensemble average of the noise. QSD reproduces the master equation in the mean:

$$
M(|\psi\rangle\langle\psi|)=\rho .
$$

(This is what is meant by an unraveling of the master equation.) Expectation values for operators obey a similar relationship:

$$
\langle\hat{O}\rangle_{\rho}=\operatorname{Tr}\{\hat{O} \rho\}=M\left(\langle\hat{O}\rangle_{\psi}\right) .
$$

The use of QSD as a practical algorithm to solve master equations has been widely investigated [5. This includes calculations of output spectra in quantum optics [6]. While it seems reasonable that there should be a relationship between output spectra and time correlation functions in QSD analogous to that in Schrödinger or master equation dynamics, this has been difficult to show, as there is no generally accepted definition of a time correlation function for a single QSD trajectory. Since QSD is framed in terms of a nonlinear stochastic differential equation for an evolving state, it is not obvious how to generalize this to products of operators at different times.

Gisin [7] has attempted to provide a Heisenberg picture for stochastically evolving operators. Sondermann [8], using the same stochastic equations, has suggested another definition for time correlation functions. In both cases it is difficult to relate this to the usual QSD formalism. In this paper we attempt to find a definition which arises from the QSD equation itself.

In section 2 we derive the output spectrum of a quantum mechanical system, and show its relationship to time correlation functions, as given by the quantum regression theorem.

In section 3 we derive a quantum output spectrum using QSD, and show how it leads to a natural definition of a two-time correlation function for a single QSD trajectory. Unlike the correlation functions which arise in the master equation derivation, these QSD functions depend explicitly on the 
measurement time and the noise. On average, however, these dependencies vanish, and the result agrees with the usual definition of a time correlation function.

In section 4 we look briefly at alternative unravelings, and discuss the use of these techniques for the practical computation of spectra. Finally, in section 5 we summarize our results and draw conclusions.

\section{Spectra and time correlation functions}

Consider a rather idealized model of an experimental set-up for measuring the output spectrum of a quantum mechanical system. Suppose we have a system with Hamiltonian $H_{1}$ and Hilbert space $\mathcal{H}_{1}$ weakly coupled to an output mode with Hamiltonian $H_{2}=\omega b^{\dagger} b$ and Hilbert space $\mathcal{H}_{2}$. We assume weak coupling to minimize the perturbation of the system evolution. For simplicity we assume a linear interaction: $H_{I}=\epsilon q\left(b+b^{\dagger}\right)$ where the operator $q$ represents a physical quantity of the system, like position for example, $b$ and $b^{\dagger}$ are the annihilation and creation operators for the output mode, and $\epsilon$ is small.

The total Hamiltonian of the system plus output mode is

$$
H=H_{1}+H_{2}+H_{I}
$$

and operates on the combined Hilbert space $\mathcal{H}_{1} \otimes \mathcal{H}_{2}$. Let the system degrees of freedom also be coupled to an environment, described by a set of environment operators $L_{m}$ acting on $\mathcal{H}_{1}$. The system plus output mode obeys the master equation (11) with Hamiltonian (6).

If the output mode is initially in the ground state, so that the initial density matrix is of the form $\rho=\rho_{00} \otimes|0\rangle\langle 0|$, we can approximate at later times

$$
\rho \approx \rho_{00} \otimes|0\rangle\left\langle 0\left|+\rho_{01} \otimes\right| 0\right\rangle\left\langle 1\left|+\rho_{10} \otimes\right| 1\right\rangle\left\langle 0\left|+\rho_{11} \otimes\right| 1\right\rangle\langle 1|+O\left(\epsilon^{3}\right),
$$

where the $\rho_{i j}$ are time-dependent operators on $\mathcal{H}_{1}$; we have explicitly separated the system and output mode degrees of freedom and neglected all but the first excited state of the output mode. We then have $\rho_{01}, \rho_{10} \sim \epsilon$ and $\rho_{11} \sim \epsilon^{2}$, and can rewrite equation (1) in the form

$$
\dot{\rho}_{00}=\mathcal{L} \rho_{00}+O\left(\epsilon^{2}\right),
$$




$$
\begin{aligned}
& \dot{\rho}_{01}=\mathcal{L} \rho_{01}+i \epsilon \rho_{00} q+i \omega \rho_{01}+O\left(\epsilon^{3}\right), \\
& \dot{\rho}_{10}=\mathcal{L} \rho_{10}-i \epsilon q \rho_{00}-i \omega \rho_{10}+O\left(\epsilon^{3}\right), \\
& \dot{\rho}_{11}=\mathcal{L} \rho_{11}-i \epsilon q \rho_{01}+i \epsilon \rho_{10} q+O\left(\epsilon^{4}\right) .
\end{aligned}
$$

$\mathcal{L}$ is the time evolution superoperator restricted to the system degrees of freedom:

$$
\mathcal{L} \rho_{i j}=-i\left[H_{1}, \rho_{i j}\right]+\sum_{m}\left(L_{m} \rho_{i j} L_{m}^{\dagger}-\frac{1}{2}\left\{L_{m}^{\dagger} L_{m}, \rho_{i j}\right\}\right) .
$$

To lowest order, $\rho_{00}$ evolves according to a normal master equation, with no reference to the output mode at all. The other components $\rho_{i j}$ represent the weak signal transmitted via the interaction with the output mode.

We can solve these equations:

$$
\begin{gathered}
\rho_{00}(t)=S_{0}^{t} \rho_{00}(0) \\
\rho_{01}(t)=i \epsilon \int_{0}^{t} S_{t^{\prime}}^{t}\left(\rho_{00}\left(t^{\prime}\right) q\right) \mathrm{e}^{i \omega\left(t-t^{\prime}\right)} d t^{\prime}=\rho_{10}^{\dagger}(t) \\
\rho_{11}(t)=-i \epsilon \int_{0}^{t} S_{t^{\prime}}^{t}\left(q \rho_{01}\left(t^{\prime}\right)\right) d t^{\prime}+\text { h.c. } \\
=\epsilon^{2} \int_{0}^{t} \int_{0}^{t^{\prime}} S_{t^{\prime}}^{t}\left(q S_{t^{\prime \prime}}^{t^{\prime}}\left(\left(S_{0}^{t^{\prime \prime}} \rho_{00}(0)\right) q\right)\right) \mathrm{e}^{i \omega\left(t^{\prime}-t^{\prime \prime}\right)} d t^{\prime \prime} d t^{\prime}+\text { h.c. }
\end{gathered}
$$

where $S_{t_{1}}^{t_{2}}=\exp \left(\mathcal{L}\left(t_{2}-t_{1}\right)\right)$ is the time evolution superoperator from time $t_{1}$ to time $t_{2}$ given by (9). The excitation of the output mode is what is measured, so we are interested in the expectation value $\left\langle b^{\dagger} b\right\rangle=\operatorname{Tr}\left\{b^{\dagger} b \rho\right\}=$ $\operatorname{Tr}\left\{\rho_{11}\right\}$. We find this by taking the trace of equation (12). Equation (11) preserves the trace, so in taking the trace of (12) the factor $\exp \left(\mathcal{L}\left(t-t^{\prime}\right)\right)$ has no effect, and may be dropped. Thus,

$$
\begin{aligned}
\left\langle b^{\dagger} b\right\rangle(t)= & \epsilon^{2} \int_{0}^{t} \int_{0}^{t^{\prime}} \operatorname{Tr}\left\{q S_{t^{\prime \prime}}^{t^{\prime}}\left(\left(S_{0}^{t^{\prime \prime}} \rho_{00}(0)\right) q\right)\right\} \mathrm{e}^{i \omega\left(t^{\prime}-t^{\prime \prime}\right)} d t^{\prime \prime} d t^{\prime}+\text { c.c. } \\
= & \epsilon^{2} \int_{0}^{t} \int_{0}^{t^{\prime}} \operatorname{Tr}\left\{q S_{t^{\prime \prime}}^{t^{\prime}}\left(\rho_{00}\left(t^{\prime \prime}\right) q\right)\right\} \mathrm{e}^{i \omega\left(t^{\prime}-t^{\prime \prime}\right)} d t^{\prime \prime} d t^{\prime}+\text { c.c. } \\
& =\epsilon^{2} \int_{0}^{t} \int_{0}^{t^{\prime}} \mathrm{e}^{i \omega\left(t^{\prime}-t^{\prime \prime}\right)}\left\langle q\left(t^{\prime}\right) q\left(t^{\prime \prime}\right)\right\rangle_{\mathrm{QRT}} d t^{\prime \prime} d t^{\prime}+\text { c.c. }
\end{aligned}
$$


where the last equality defines the two-time correlation function $\left\langle q\left(t^{\prime}\right) q\left(t^{\prime \prime}\right)\right\rangle_{\mathrm{QRT}}$. Note that $\left\langle q\left(t^{\prime}\right) q\left(t^{\prime \prime}\right)\right\rangle_{\mathrm{QRT}}$ is determined by the evolution operator $S_{0}^{t}$ of the reduced mixed state $\rho_{00}$, in accordance with the quantum regression theorem [2]. We see that the expected output has the form of a Fourier transform of a two-time correlation function; this is just like the result in classical physics [9]. Examining output modes at different frequencies $\omega$ gives the spectrum of the system.

\section{$3 \quad$ Spectra and time correlations in QSD}

We can calculate the results of section 2 with QSD by using relation (5). We solve the QSD equation (2) for a state in the combined Hilbert space $\mathcal{H}=\mathcal{H}_{1} \otimes \mathcal{H}_{2}$, starting with an initial condition $|\Psi\rangle=\left|\psi_{0}\right\rangle|0\rangle$. By averaging $\left\langle b^{\dagger} b\right\rangle_{\Psi(t)}$ over many trajectories we reproduce (13). This was done in [6] for the case of second harmonic generation.

How can we interpret $\left\langle b^{\dagger} b\right\rangle_{\Psi(t)}$ for a single trajectory? Let's examine a little more closely the evolution (2). We can separate the components of $\Psi$

$$
|\Psi(t)\rangle=\left|\phi_{0}(t)\right\rangle|0\rangle+\left|\phi_{1}(t)\right\rangle|1\rangle+O\left(\epsilon^{2}\right),
$$

where we again neglect all excited states of the output mode above the first. The initial condition is

$$
\left|\phi_{0}(0)\right\rangle=\left|\psi_{0}\right\rangle, \quad\left|\phi_{1}(0)\right\rangle=0 \text {. }
$$

The QSD equation becomes a pair of coupled equations

$$
\begin{aligned}
d\left|\phi_{0}\right\rangle & =-i H_{1}\left|\phi_{0}\right\rangle d t+\sum_{m}\left(\left\langle L_{m}^{\dagger}\right\rangle_{\phi_{0}} L_{m}-\frac{1}{2} L_{m}^{\dagger} L_{m}-\frac{1}{2}\left\langle L_{m}^{\dagger}\right\rangle_{\phi_{0}}\left\langle L_{m}\right\rangle_{\phi_{0}}\right)\left|\phi_{0}\right\rangle d t \\
& +\sum_{m}\left(L_{m}-\left\langle L_{m}\right\rangle_{\phi_{0}}\right)\left|\phi_{0}\right\rangle d \xi_{m}+O\left(\epsilon^{2}\right), \\
d\left|\phi_{1}\right\rangle \quad & =-i H_{1}\left|\phi_{1}\right\rangle d t+\sum_{m}\left(\left\langle L_{m}^{\dagger}\right\rangle_{\phi_{0}} L_{m}-\frac{1}{2} L_{m}^{\dagger} L_{m}-\frac{1}{2}\left\langle L_{m}^{\dagger}\right\rangle_{\phi_{0}}\left\langle L_{m}\right\rangle_{\phi_{0}}\right)\left|\phi_{1}\right\rangle d t \\
& +\sum_{m}\left(L_{m}-\left\langle L_{m}\right\rangle_{\phi_{0}}\right)\left|\phi_{1}\right\rangle d \xi_{m}-i \omega\left|\phi_{1}\right\rangle d t-i \epsilon q\left|\phi_{0}\right\rangle d t+O\left(\epsilon^{3}\right),
\end{aligned}
$$


Note that (16) is identical to the usual QSD equation for the system alone to first order in $\epsilon$. Just as in section 2, this approximation gains an extra order of $\epsilon$ in accuracy for free. Thus, the system degrees of freedom have the usual quantum state diffusion behavior, essentially uninfluenced by the interaction with the output mode. Exactly as in (8), we have an almost unperturbed system, with a weak signal transmitted to the outside world.

Equation (17) is interesting, in that all of the expectation values in this equation are calculated with respect to $\left|\phi_{0}\right\rangle$, so this is a driven linear equation with time-dependent coefficients. Given the solution $\left|\phi_{0}(t)\right\rangle$, we can find $\left|\phi_{1}(t)\right\rangle$, at least in principle.

We formally integrate (16) to get

$$
\left|\phi_{0}\left(t_{2}\right)\right\rangle=T\left(\xi, \psi_{0}\right)_{t_{1}}^{t_{2}}\left|\phi_{0}\left(t_{1}\right)\right\rangle
$$

where $T\left(\xi, \psi_{0}\right)_{t_{1}}^{t_{2}}$ is the time-evolution operator from time $t_{1}$ to time $t_{2}$. It explicitly depends on the noise $\xi$ and the initial state $\left|\psi_{0}\right\rangle$, since the QSD equation is nonlinear. Given this time-evolution operator, the solution to (17) is

$$
\left|\phi_{1}(t)\right\rangle=-i \epsilon \int_{0}^{t} T\left(\xi, \psi_{0}\right)_{t^{\prime}}^{t} q\left|\phi_{0}\left(t^{\prime}\right)\right\rangle \mathrm{e}^{-i \omega\left(t-t^{\prime}\right)} d t^{\prime} .
$$

The output spectrum is then

$$
\left\langle b^{\dagger} b\right\rangle_{\Psi}(t)=\epsilon^{2} \int_{0}^{t} \int_{0}^{t}\left\langle\psi_{0}\left|T_{0}^{\dagger t^{\prime}} q T_{t^{\prime}}^{\dagger} T_{t^{\prime \prime}}^{t} q T_{0}^{t^{\prime \prime}}\right| \psi_{0}\right\rangle \mathrm{e}^{i \omega\left(t^{\prime}-t^{\prime \prime}\right)} d t^{\prime} d t^{\prime \prime}
$$

where the $\xi$ and $\psi_{0}$ have been suppressed for conciseness. This expression resembles a Fourier transform of some kind of correlation function, just as was the case in section 2; but this correlation function is defined for a single QSD trajectory.

We can bring this rather closer to the treatment in section 2 by introducing the projector

$$
\begin{aligned}
P_{\Psi} & =|\Psi\rangle\langle\Psi|, \\
& =P_{00}|0\rangle\left\langle 0\left|+P_{01}\right| 0\right\rangle\left\langle 1\left|+P_{10}\right| 1\right\rangle\left\langle 0\left|+P_{11}\right| 1\right\rangle\langle 1|,
\end{aligned}
$$

where these partial projectors $P_{i j}$ are operators on $\mathcal{H}_{1}$. These are related to our earlier treatment by

$$
P_{00}=\left|\phi_{0}\right\rangle\left\langle\phi_{0}\left|, \quad P_{01}=\right| \phi_{0}\right\rangle\left\langle\phi_{1}\left|, \quad P_{10}=\right| \phi_{1}\right\rangle\left\langle\phi_{0}\left|, \quad P_{11}=\right| \phi_{1}\right\rangle\left\langle\phi_{1}\right|,
$$


and to section 2 by

$$
\rho_{i j}=M\left(P_{i j}\right) .
$$

These $P_{i j}$ are not themselves projectors; however, $P_{00}^{2}=P_{00}+O\left(\epsilon^{2}\right)$, and therefore can be considered a projector to good approximation.

We define a time evolution superoperator for these partial projectors

$$
S\left(\xi, \psi_{0}\right)_{t_{1}}^{t_{2}} P=T\left(\xi, \psi_{0}\right)_{t_{1}}^{t_{2}} P T^{\dagger}\left(\xi, \psi_{0}\right)_{t_{1}}^{t_{2}}
$$

This is related to the superoperator for the master equation by

$$
M\left(S\left(\xi, \psi_{0}\right)_{t_{1}}^{t_{2}}\right) P_{00}=S_{t_{1}}^{t_{2}} P_{00}=\exp \left(\mathcal{L}\left(t_{2}-t_{1}\right)\right) P_{00} .
$$

We shall need the following solutions:

$$
\begin{gathered}
P_{00}(t)=S\left(\xi, \psi_{0}\right)_{0}^{t} P_{00}(0), \\
P_{01}(t)=i \epsilon \int_{0}^{t} S\left(\xi, \psi_{0}\right)_{t^{\prime}}^{t}\left(P_{00}\left(t^{\prime}\right) q\right) \mathrm{e}^{i \omega\left(t-t^{\prime}\right)} d t^{\prime}=P_{10}^{\dagger}(t)
\end{gathered}
$$

In order to compute the output signal $\left\langle b^{\dagger} b\right\rangle_{\psi(t)}$ we make use of the equality:

$$
P_{11}(t)=P_{10} P_{01} .
$$

In this way, we obtain the following expression for the output signal:

$$
\begin{gathered}
\left\langle b^{\dagger} b\right\rangle_{\psi(t)}=\operatorname{Tr}\left\{P_{10} P_{01}\right\} \\
=\epsilon^{2} \int_{0}^{t} d t^{\prime} \operatorname{Tr}\left\{S\left(\xi, \psi_{0}\right)_{t^{\prime}}^{t}\left(P_{00}\left(t^{\prime}\right) q\right)^{\dagger} \int_{0}^{t} d t^{\prime \prime} S\left(\xi, \psi_{0}\right)_{t^{\prime \prime}}^{t}\left(P_{00}\left(t^{\prime \prime}\right) q\right)\right\} \mathrm{e}^{i \omega\left(t^{\prime}-t^{\prime \prime}\right)}, \\
=\epsilon^{2} \int_{0}^{t} d t^{\prime} \operatorname{Tr}\left\{T_{t^{\prime}}^{t} q P_{00}\left(t^{\prime}\right) T_{t^{\prime}}^{t^{\dagger}} \int_{0}^{t} d t^{\prime \prime} T_{t^{\prime \prime}}^{t} P_{00}\left(t^{\prime \prime}\right) q T_{t^{\prime \prime}}^{t}\right\} \mathrm{e}^{i \omega\left(t^{\prime}-t^{\prime \prime}\right)} .
\end{gathered}
$$

Note that the two central operators $T_{t^{\prime}}^{t \dagger} T_{t^{\prime \prime}}^{t}$ can not be replaced with $T_{t^{\prime}}^{t^{\prime \prime}}$.

Accordingly, in analogy with (13) we define a measurement-dependent two-time correlation function for QSD:

$$
C\left(\hat{O}_{2}, t_{2} ; \hat{O}_{1}, t_{1} ; t\right) \equiv \operatorname{Tr}\left\{S\left(\xi, \psi_{0}\right)_{t_{2}}^{t}\left(\hat{O}_{2} P_{00}\left(t_{2}\right)\right) S\left(\xi, \psi_{0}\right)_{t_{1}}^{t}\left(P_{00}\left(t_{1}\right) \hat{O}_{1}\right)\right\}
$$

Note that this assumes $t_{1}<t_{2}$; for $t_{1}>t_{2}$ an analogous expression can be formed. This function $C$ in QSD has the nice feature that it is the trace of a 
product of two operators, each involving one of the times $t_{1}$ and $t_{2}$, similar to correlation functions of classical stochastic processes (which are products of the random variables at different times [9]). However, it also has the strange feature that the "final" time $t$ appears in this definition. This is why we term $C$ a measurement-dependent correlation function, rather than a true correlation function. Note that in perfect analogy with the quantum regression theorem, $C\left(\hat{O}_{2}, t_{2} ; \hat{O}_{1}, t_{1} ; t\right)$ is determined by the evolution operator $S\left(\xi, \psi_{0}\right)_{t_{1}}^{t_{2}}$ of the reduced system's pure state $\left|\phi_{0}(t)\right\rangle$.

It remains to establish the relation between the function $C\left(\hat{O}_{2}, t_{2} ; \hat{O}_{1}, t_{1} ; t\right)$ and the QRT correlation function introduced in section 2. For this purpose we note that $P_{11}=\left|\phi_{1}\right\rangle\left\langle\phi_{1}\right|$ provides an equivalent expression for the output signal:

$$
\begin{gathered}
\left\langle b^{\dagger} b\right\rangle_{\Psi}(t)=\operatorname{Tr}\left\{P_{11}\right\} \\
=\epsilon^{2} \int_{0}^{t} \int_{0}^{t^{\prime}} \operatorname{Tr}\left\{S\left(\xi, \psi_{0}\right)_{t^{\prime}}^{t}\left(q S\left(\xi, \psi_{0}\right)_{t^{\prime \prime}}^{t^{\prime}}\left(P_{00}\left(t^{\prime \prime}\right) q\right)\right)\right\} \mathrm{e}^{i \omega\left(t^{\prime}-t^{\prime \prime}\right)} d t^{\prime} d t^{\prime \prime}+\text { c.c. }
\end{gathered}
$$

Using this relation (31) we can derive an equivalent form of the function $C$ :

$$
\begin{gathered}
C\left(\hat{O}_{2}, t_{2} ; \hat{O}_{1}, t_{1} ; t\right)= \\
\operatorname{Tr}\left\{S\left(\xi, \psi_{0}\right)_{t_{2}}^{t}\left(\hat{O}_{2} S\left(\xi, \psi_{0}\right)_{t_{1}}^{t_{2}}\left(P_{00}\left(t_{1}\right) \hat{O}_{1}\right)\right)\right\},
\end{gathered}
$$

Note that in section 2 it was possible to remove the time evolution superoperator $S_{t^{\prime}}^{t}$, as it did not affect the trace. This is not true in the single trajectory case, as explained above; so the function $C$ depends explicitly on time, as mentioned above. Nevertheless, taking the mean over the noise of $C\left(\hat{O}_{2}, t_{2} ; \hat{O}_{1}, t_{1} ; t\right)$, one recovers the correlation function derived for mixed states from the quantum regression theorem:

$$
M\left(C\left(\hat{O}_{2}, t_{2} ; \hat{O}_{1}, t_{1} ; t\right)\right)=\left\langle\hat{O}_{2}\left(t_{2}\right) \hat{O}_{1}\left(t_{1}\right)\right\rangle_{\mathrm{QRT}} .
$$

Note that the dependence on the final time $t$ vanishes in the mean.

While the definition (30) arises naturally in this derivation, and has the correct average behavior, its dependence on $t$ remains a puzzling and rather annoying feature. It is possible to make a different definition, closely related to that of (30), which avoids this problem. Since the operator $S\left(\xi, \psi_{0}\right)_{t_{2}}^{t}$ in the definition (32) vanishes in the mean, we can define a true two-time correlation function:

$$
\left\langle\hat{O}_{2}\left(t_{2}\right) \hat{O}_{1}\left(t_{1}\right)\right\rangle_{\mathrm{QSD}} \equiv \operatorname{Tr}\left\{\hat{O}_{2} S\left(\xi, \psi_{0}\right)_{t_{1}}^{t_{2}}\left(P_{00}\left(t_{1}\right) \hat{O}_{1}\right)\right\}
$$


In this way the "final time" $t$ disappears and the correlation function is formally identical to the QRT case (but for vectors instead of matrices). Moreover, the correlation function then appears as a scalar product of two vectors:

$$
\left\langle\hat{O}_{2}\left(t_{2}\right) \hat{O}_{1}\left(t_{1}\right)\right\rangle_{\mathrm{QSD}}=\left\langle T_{t_{1}}^{t_{2}} \hat{O}_{1} \phi_{0}\left(t_{1}\right) \mid \hat{O}_{2} \phi_{0}\left(t_{2}\right)\right\rangle .
$$

One can consider this new definition to be an average over "future" noise, i.e., noise after $t_{2}$.

$$
\left\langle\hat{O}_{2}\left(t_{2}\right) \hat{O}_{1}\left(t_{1}\right)\right\rangle_{\mathrm{QSD}}=M\left(C\left(\hat{O}_{2}, t_{2} ; \hat{O}_{1}, t_{1} ; t\right)\right)_{\xi\left(t^{\prime}\right), t_{1}<t_{2}<t^{\prime}<t} .
$$

Certainly, this once again reproduces the QRT correlation function in the mean, and gives exactly the same $\left\langle b^{\dagger} b\right\rangle_{\psi(t)}$ for a single trajectory:

$$
M\left(\left\langle\hat{O}_{2}\left(t_{2}\right) \hat{O}_{1}\left(t_{1}\right)\right\rangle_{\mathrm{QSD}}\right)=\left\langle\hat{O}_{2}\left(t_{2}\right) \hat{O}_{1}\left(t_{1}\right)\right\rangle_{\mathrm{QRT}}
$$

While this definition removes the dependence on the final time $t$, this two-time correlation function still has some interesting features, related to the fact that the QSD time-evolution superoperator (24) does not preserve the trace. In particular, we note that if the second operator $\hat{O}_{2}$ is the identity $\hat{I}$, then

$$
\left\langle\hat{I}\left(t_{2}\right) \hat{O}_{1}\left(t_{1}\right)\right\rangle_{\mathrm{QSD}} \neq\left\langle\hat{O}_{1}\left(t_{1}\right)\right\rangle
$$

contrary to the case of the QRT. If $\hat{O}_{1}=\hat{I}$ no such difficulty arises. Since unravelings of the master equation do not generically preserve the trace, this feature will arise in any similar derivation. This might be one argument for using an unraveling specifically chosen to preserve the trace, such as that used by Gisin in his discussion of a Heisenberg picture for QSD [7, 8]. In any case, the desired relation does hold in the mean:

$$
M\left(\left\langle\hat{I}\left(t_{2}\right) \hat{O}_{1}\left(t_{1}\right)\right\rangle_{\mathrm{QSD}}\right)=M\left(\left\langle\hat{O}_{1}\left(t_{1}\right)\right\rangle\right) .
$$

Fortunately, correlation functions of the above form would not arise in any physically reasonable measurement scheme, since $\hat{I}$ does not describe an interaction.

The definition of the two-time correlation function (34), and its relation to the quantum regression theorem (37) are the main results of this article. The central line of arguments can be summarized as follows:

$$
\rho_{11}=M\left(P_{11}\right)=M\left(P_{10} P_{01}\right) \neq M\left(P_{10}\right) M\left(P_{01}\right)=\rho_{10} \rho_{01}
$$




\section{Alternative unravelings and practical cal- culations}

While the definition (34) arises naturally from the QSD analysis of this problem, it is not the only possible definition of a time correlation function for a single trajectory; indeed, there are an infinite number of such definitions, corresponding to different unravelings [10].

One such alternative unraveling has already been proposed by Gisin as a possible definition of a time-correlation function for individual trajectories, as well as providing a sort of Heisenberg picture corresponding to QSD [7]. Here we will suggest yet another such alternative unraveling.

In particular, rather than solving the Lindblad master equation itself and then solving for the output spectrum, one might instead begin with the expression (13) and attempt to unravel the time-correlation function directly.

What form would such an unraveling take? In QSD, one unravels the density operator evolution into many trajectories, each consisting of a single state, and with a mean $\rho=M(|\psi\rangle\langle\psi|)$. This works because $\rho$ is hermitian: $\rho=\rho^{\dagger}$. For a time correlation function, this is no longer sufficient. The form given by the quantum regression theorem is

$$
\left\langle q\left(t_{2}\right) q\left(t_{1}\right)\right\rangle_{\mathrm{QRT}}=\operatorname{Tr}\left\{q S_{t_{1}}^{t_{2}}\left(\rho\left(t_{1}\right) q\right)\right\},
$$

where $S_{t_{1}}^{t_{2}}$ is the time-evolution superoperator defined by the master equation (苗); however, the "initial state" $\rho\left(t_{1}\right) q$ is not hermitian. Therefore the evolution from $t_{1}$ to $t_{2}$ cannot be unraveled in terms of pure states.

One can, however, consider a pair of vectors $|\psi\rangle$ and $|\phi\rangle$ such that $M(|\psi\rangle\langle\phi|)$ does reproduce the correct evolution. Such a diad equation is quite analogous to the usual QSD equation.

One pair of coupled equations that do the job are

$$
\begin{aligned}
|d \psi\rangle & =\left(-i H+\left\langle L^{\dagger}\right\rangle_{\phi} L-\frac{1}{2} L^{\dagger} L-\frac{1}{2}\left\langle L^{\dagger}\right\rangle_{\phi}\langle L\rangle_{\psi}\right)|\psi\rangle d t+\left(L-\langle L\rangle_{\psi}\right)|\psi\rangle d \xi, \\
\langle d \phi| & =\langle\phi|\left(i H+L^{\dagger}\langle L\rangle_{\psi}-\frac{1}{2} L^{\dagger} L-\frac{1}{2}\left\langle L^{\dagger}\right\rangle_{\phi}\langle L\rangle_{\psi}\right) d t+\langle\phi|\left(L^{\dagger}-\left\langle L^{\dagger}\right\rangle_{\phi}\right) d \xi^{*} .
\end{aligned}
$$

It is not hard to show that

$$
M(d|\psi\rangle\langle\phi|)=\mathcal{L}(|\psi\rangle\langle\phi|),
$$


so this has the correct evolution in the mean. If $|\psi\rangle=|\phi\rangle$ then these coupled equations reduce to the ordinary QSD equation (2). Note that in general, however, the normalization of $|\psi\rangle$ and $|\phi\rangle$ is not preserved.

The technique for calculating time correlation functions is as follows. If the initial density matrix is a pure state, $\rho(0)=\left|\psi_{0}\right\rangle\left\langle\psi_{0}\right|$, then one begins with both states equal $|\psi\rangle=|\phi\rangle=\left|\psi_{0}\right\rangle$, and evolves them to time $t_{1}$ according to (42), which is equivalent to the QSD equation (2). At time $t_{1}$, multiply $\left\langle\phi\left(t_{1}\right)\right| \rightarrow\left\langle\phi\left(t_{1}\right)\right| q$ and continue to evolve the diad according to (42). At time $t_{2}$, multiply $\left|\psi\left(t_{2}\right)\right\rangle \rightarrow q\left|\psi\left(t_{2}\right)\right\rangle$ and take the trace. The mean over many such trajectories equals the time-correlation function (41).

This pair of equations (42) shares many properties in common with the QSD equation (2), but unlike QSD is not uniquely defined. Since only the composite diad $|\psi\rangle\langle\phi|$ is important, the norm and phase can be shifted arbitrarily between these two states. Diósi has likened this to a gauge freedom [10]. Many such pairs of equations are therefore possible, as well as others with properties radically different from QSD; just as QSD is one of many unravelings of the master equation, albeit with unique symmetry properties. To solve for output spectra, of course, one must still Fourier transform the calculated time correlation functions.

In fact, one can see that there are two distinct approaches to computing output spectra using QSD. One is to use the definition (34) or alternative definitions such as (42) to calculate the time-correlation function, averaging over many runs, and taking the Fourier transform (13).

Alternatively, one can solve the QSD equation for the entire system plus output mode; the state is then in the larger Hilbert space $\mathcal{H}_{1} \otimes \mathcal{H}_{2}$. This is essentially the approach taken by Schack et al. [6], who have also shown that these techniques can be used to calculate other quantities of interest, such as the spectrum of squeezing.

Both approaches appear to have their advantages and disadvantages, and to be roughly equal in computational difficulty. It is likely that the best approach will vary from problem to problem.

Note also that if we had unraveled the master equation (11) using an unraveling other than QSD, an exactly analogous argument would have followed. We would be able to resolve the equation for the system plus output mode into a pair of coupled equations, one corresponding to the unperturbed evolution of the system alone, the other to an output signal completely driven by the system. Thus, this type of argument could be used to define a notion 
of time correlation functions for single trajectories in any unraveling, such as the Quantum Monte Carlo techniques or the orthojumps of Diósi [4, 11, 12].

These Quantum Monte Carlo (or Quantum Jump) techniques deserve further comment, as there have already been a number of papers published on their use in the calculation of time correlation functions [13, 14, 15]. These are relevant to our current discussion, since it can be shown that the equations for Quantum Jumps become identical to those for QSD in the case of heterodyne measurements [16].

The treatment of Gardiner, Parkins, and Zoller [14 is particularly interesting in this context. Their definition of the time correlation function involves defining an auxiliary vector $|\beta, t\rangle$ which is driven by the evolution of the quantum jump vector $|\phi, t\rangle$, but does not in turn affect it.

As stated in that paper [14] their equations are rather different. In particular, they are considering only real noise, and have not gone to the weak coupling limit we have assumed in this paper, where the output has a negligibly weak effect on the system over short times. Wiseman and Milburn [16] have generalized this treatment to consider the case of heterodyne measurements, introducing complex noise and showing that this limit is exactly equivalent to the Quantum State Diffusion equation. In the limit of heterodyne measurement with weak coupling to the external mode, the equation for $|\phi, t\rangle$ becomes the QSD equation (2), and the two vectors $|\phi, t\rangle$ and $|\beta, t\rangle$ obey a pair of coupled equations identical to (16) and (17). The output spectrum is given by the mean of $\langle\beta, t \mid \beta, t\rangle$, just as in (20). From this, one could follow an argument exactly analogous to that of section 3 in this paper to arrive at a definition of a two-time correlation function identical to (34).

\section{Conclusions}

In calculating quantum optical spectra, a common approach is to calculate the quantum time correlation function and derive the spectrum by taking its Fourier transform. This time correlation function has a form given by the quantum regression theorem, and requires a solution of the master equation.

Quantum state diffusion provides in many cases an efficient method of solving the master equation. But hitherto, an appropriate definition of the time correlation function for a single QSD trajectory has been lacking, making QSD less useful for the calculation of spectra. 
In this paper we have derived such a definition in a straightforward way, quite analogous to the derivation in the case of the full master equation. This correlation function in the mean has the form given by the quantum regression theorem, and the equations for it are very close to the original QSD equation, up to $O\left(\epsilon^{2}\right)$ in the interaction strength.

This definition can be used as a practical numerical tool in computing quantum optical spectra. Other possible definitions and their potential for practical use have been briefly discussed.

Finally, let us discuss the meaning of our result in the simple case of a damped harmonic oscillator at zero temperature. The stationary solution is the ground state. Hence, once the system has reached this state, nothing happens, and it is clear that the spectrum of the damped oscillator is not contained in the evolution of its state vector. Nevertheless, the quantum regression theorem tells us that all spectra, in particular the one corresponding to the position fluctuation, are contained in the evolution operator for the corresponding master equation. Similarly, the results presented here tell us that the spectra are also contained in the stochastic evolution operator $T\left(\xi, \psi_{0}\right)_{t_{1}}^{t_{2}}$ of the QSD description of the damped oscillator. The physics behind this is that whenever a spectrum is measured, the system's environment is changed, hence its dynamics is perturbed. For example, to measure the spectrum of position fluctuations, something like weak position measurements have to be applied, and the ground state is no longer stationary [17]. However, in contrast to standard quantum measurements, this perturbation can be made arbitrarily small (corresponding to small amplitudes of the measured spectrum) over an arbitrarily long period of time. To first order, the system's evolution is unaffected, but its states acts like a source for the signal, as reflected by our equations (16) and (17). Hence the unaffected evolution operator (Liouville operator or QSD propagator or other stochastic propagator 4,13 ) contains the information about spectra that cannot actually be measured without affecting (i.e., weakly perturbing) the system's evolution.

\section{Acknowledgments}

We would like to acknowledge many useful conversations with Lajos Diósi, Ian Percival, Marco Rigo, Rüdiger Schack, Walter Strunz and Tim Spiller. We would also like to thank our referee, who provided a great deal of impor- 
tant feedback and helped us to clarify our results. This work was supported in part by the Swiss National Science Foundation.

\section{References}

[1] B.R. Mollow, Phys. Rev., 188, 1969 (1969).

[2] M. Lax, Phys. Rev., 157, 213 (1967).

[3] C.W. Gardiner Quantum Noise, Springer, Berlin, 1991.

[4] H. Carmichael, An Open Systems Approach to Quantum Optics, Springer, 1994.

[5] N. Gisin, I.C. Percival, J. Phys. A, 25, 5677 (1992); N. Gisin, I.C. Percival, J. Phys. A, 26, 2233 (1993); N. Gisin, I.C. Percival, J. Phys. A, 26, 2245 (1993); R. Schack, T.A. Brun, I.C. Percival, J. Phys. A, 28, 5401 (1995).

[6] R. Schack, T.A. Brun, I.C. Percival, to appear in Phys. Rev. A.

[7] N. Gisin, J. Mod. Optics, 40, 2313 (1993).

[8] D.G. Sondermann, J. Mod. Optics, 42, 1659 (1995).

[9] W. Horsthemke and R. Lefever, Noise induced transitions, Springer, Berlin, 1984.

[10] L. Diósi, private communication.

[11] J. Dalibard, Y. Castin, K. Molmer, Phys. Rev. Lett., 68, 580 (1992).

[12] L. Diósi, Phys. Lett. A, 114, 451 (1986). L. Diósi, Phys. Lett. A, 185, 5 (1994).

[13] K. Møllmer, Y. Castin, J. Dalibard, J. Opt. Soc. Am. B, 10, 525 (1993).

[14] C.W. Gardiner, A.S. Parkins, P. Zoller, Phys. Rev. A, 46, 4363 (1992).

[15] R. Dum, A.S. Parkins, P. Zoller, C.W. Gardiner, Phys. Rev. A, 46, 4382 (1992). 
[16] H.M. Wiseman, G.J. Milburn, Phys. Rev. A, 47, 1652 (1993). P.L. Knight, B.M. Garraway, Proc. 44th Scottish Universities Summer School in Physics, Institute of Physics Publishing, Bristol (1995).

[17] Y. Salama, N. Gisin, Phys. Lett. A, 181, 269 (1993).

Figure 1. The interaction between a quantum system with Hamiltonian $H_{1}$ interacting with an external field mode $H_{2}=\omega b^{\dagger} b$ via an interaction potential $H_{I}=\epsilon q\left(b^{\dagger}+b\right)$. The coupling of the system to the environment is modeled by a single environment operator $L$. Dotted lines represent Hamiltonian terms and dashed lines represent coupling to the environment. 

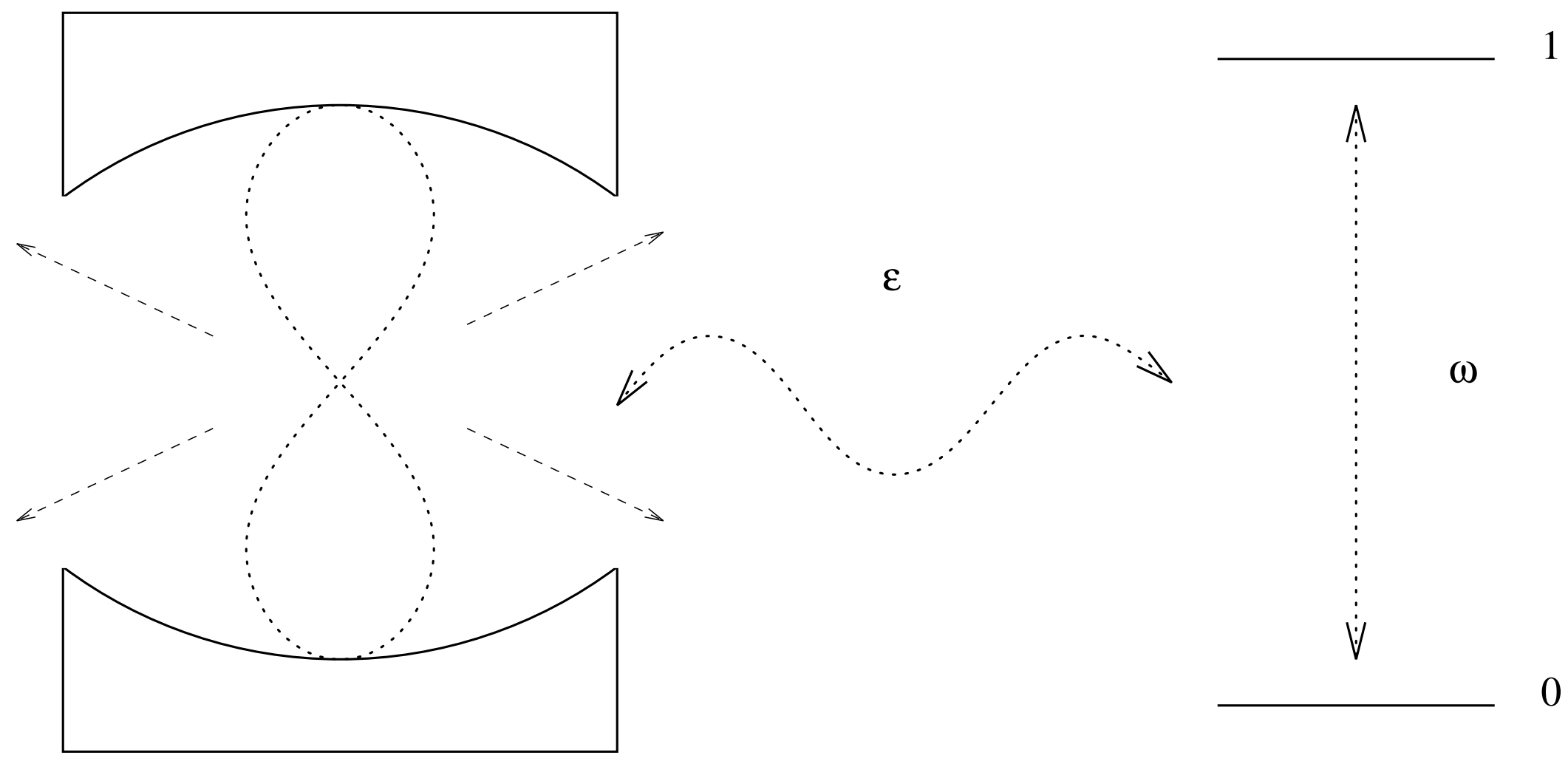

$$
H_{1}, L
$$

Fig. 1 